\title{
Response of Meningeal Carcinomatosis from Breast Cancer to Capecitabine Monotherapy: A Case Report
}

\author{
Yumi Tanaka Shoji Oura Tatsuya Yoshimasu \\ Fuminori Ohta Koma Naito Rie Nakamura \\ Yoshimitsu Hirai Masako Ikeda Yoshitaka Okamura
}

Department of Thoracic and Cardiovascular Surgery, Wakayama Medical University, Wakayama, Japan

\section{Key Words}

Breast cancer $\cdot$ Capecitabine $\cdot$ Meningeal carcinomatosis

\begin{abstract}
A 62-year-old woman with breast cancer received neoadjuvant chemotherapy followed by breast-conserving surgery and sentinel node biopsy. During adjuvant endocrine therapy with aromatase inhibitor, she developed multiple bone metastases. Thereafter, she received tamoxifen and zoledronate therapy. In May 2011, she developed a tongue deviation and was diagnosed as having meningeal carcinomatosis. The tongue deviation disappeared 3 weeks after taking capecitabine $(2,400 \mathrm{mg} /$ day). Magnetic resonance imaging of the brain showed regression of meningeal carcinomatosis. Levels of tumor markers CEA and CA15-3 changed from $96.0 \mathrm{lU} / \mathrm{ml}$ and $3.5 \mathrm{ng} / \mathrm{ml}$ to $47.0 \mathrm{IU} / \mathrm{ml}$ and $1.5 \mathrm{ng} / \mathrm{ml}$, respectively. Progression-free survival with capecitabine monotherapy was 5 months.
\end{abstract}

\section{Introduction}

Meningeal carcinomatosis (MC) is a rare clinical manifestation among metastatic solid tumors. Malignant cells infiltrate the subarachnoid space and cerebral pia mater, causing meningeal irritation and cranial nerve symptoms. MC is frequently diagnosed in patients with breast cancer, lung cancer, lymphoma or leukemia as well as tumors of the central nervous system (CNS), mostly medulloblastoma [1]. Prognosis for MC patients is poor, with a median survival of $2-4$ months. Intrathecal chemotherapy is effective in patients with MC from lymphoma, leukemia and breast cancer [2]. However, 
intrathecal treatment requires invasive procedures, preferably repeated lumber puncture, and limits the use of chemotherapy agents due to the cerebrospinal damage.

We report here a breast cancer patient with MC who responded to capecitabine monotherapy.

\section{Case Report}

A 60-year-old postmenopausal woman was referred to our hospital due to the tumor in the right breast, in April 2008. She was diagnosed with stage IIB breast cancer (cT3N0M0, invasive lobular carcinoma), and received 12 cycles of weekly paclitaxel $80 \mathrm{mg} / \mathrm{m}^{2}$, with FEC (5-fluorouracil 500 $\mathrm{mg} / \mathrm{m}^{2}$, epirubicin $100 \mathrm{mg} / \mathrm{m}^{2}$, cyclophosphamide $500 \mathrm{mg} / \mathrm{m}^{2}$ ) as a neoadjuvant chemotherapy (NAC). The tumor showed a clinical partial response to NAC. She underwent breast-conserving surgery and sentinel lymph node biopsy in October 2008. Pathological examination showed the breast cancer to be node-negative, estrogen receptor-positive (99\%), progesterone receptor-negative, and human epidermal growth factor receptor type 2 (HER2)-negative. Chemotherapeutic effect of the NAC was grade $1 \mathrm{~b}$ [3]. After surgery, she received adjuvant radiation therapy and exemestane. In December 2010, positron emission tomography revealed multiple bone metastases. Thereafter, she received zoledronic acid and tamoxifen. In May 2011, she developed a tongue deviation; magnetic resonance imaging (MRI) showed multiple leptomeningeal metastases (fig. 1). Serum cancer antigen 15-3 (CA15-3) and carcinoembryonic antigen (CEA) levels increased from $7.0 \mathrm{IU} / \mathrm{ml}$ and $0.3 \mathrm{ng} / \mathrm{ml}$ to $96.0 \mathrm{IU} / \mathrm{ml}$ and $3.5 \mathrm{ng} / \mathrm{ml}$, respectively. Cerebrospinal fluid (CSF) analysis was not performed; MC was diagnosed through positive imaging and neurological symptoms. Based on the diagnosis of $\mathrm{MC}$, she received capecitabine monotherapy $(2,400 \mathrm{mg} /$ day). Only 3 weeks later, her tongue deviation disappeared. Since she had started receiving capecitabine, CA15-3 and CEA levels gradually reduced (fig. 2), and MRI showed reductions of the leptomeningeal lesions (fig. 3). Side effects of capecitabine were negligible except for grade 1 hand-foot syndrome. Progression-free survival with capecitabine monotherapy was 5 months.

\section{Discussion}

In America and European countries, breast cancer is most frequently associated with MC among extra-CNS solid malignancies. About 2-5\% of patients with breast cancer develop MC, usually late in the course of their metastatic disease [4]. On the other hand, in Japan, gastric cancer (55\%) and lung cancer (30\%) are highly associated with MC, but MC from breast cancer is extremely rare $(1.7 \%)$ [5]. There are no distinct reasons for this difference, which may be attributed to the lower disease rate of breast cancer itself, or the lower incidence of invasive lobular carcinoma of all histologic subtypes of breast cancer in Japan. Gauthier et al. [6] reported a high incidence (28\%) of invasive lobular carcinoma in MC from breast cancer $[2,7]$.

By hematogenous spread or direct extension from bone metastasis or brain metastasis, tumor cells reach the leptomeninges and disseminate throughout the CNS through CSF flow. The most common sign is multiple cranial nerve palsies, but headache, backache, polyradiculopathies, incontinence, confusional state, lower motor neuron weakness and sensory abnormalities may be the principal manifestations $[4,8]$. This patient had multiple bone metastases and developed hypoglossal nerve palsy.

Although MC from breast cancer shows the best prognosis among $\mathrm{MC}$ from various malignancies, median overall survival is no more than 6 months following diagnosis of MC from breast cancer [9]. Treatment of MC relies mainly on intrathecally and/or systemically delivered chemotherapy, combined with other treatment modalities, such 
as irradiation or debulking surgery of meningeal metastases. There are no standard chemotherapy regimens for MC because neither prospective nor retrospective studies have shown any anticancer drugs to be highly effective against MC.

There are reports of breast cancer-derived MC that responded to hormonal manipulation alone $[2,8]$, and some cases that demonstrate the efficacy of intrathecal trastuzumab against MC from HER2-positive breast cancer $[10,11]$. The present case was hormone-resistant and HER2-negative breast cancer, which limited options for chemotherapy.

There is only 1 case report describing the efficacy of capecitabine monotherapy against MC from breast cancer [12]. Capecitabine is an oral prodrug of fluorouracil used in the palliative treatment of metastatic breast cancer that is resistant to taxane and anthracycline chemotherapy. Animal studies demonstrate that capecitabine and its metabolites cross the blood-brain barrier in limited quantities [13]. A study in humans using a fluoropyrimidine similar to capecitabine found that fluorouracil concentrations in CSF were only 1-3\% of plasma concentrations [14]. We do not know the mechanism of response to capecitabine in our case, but a similar rapid and durable response to capecitabine was reported in a patient with refractory brain metastasis from breast cancer [15]. It is possible that diffuse MC in our case disrupted the blood-CSF barrier and allowed a higher fluorouracil concentration in the CSF. Since most patients with MC from breast cancer have various prior therapies and poor performance status, capecitabine monotherapy is a feasible approach in the treatment of $\mathrm{MC}$ from breast cancer.

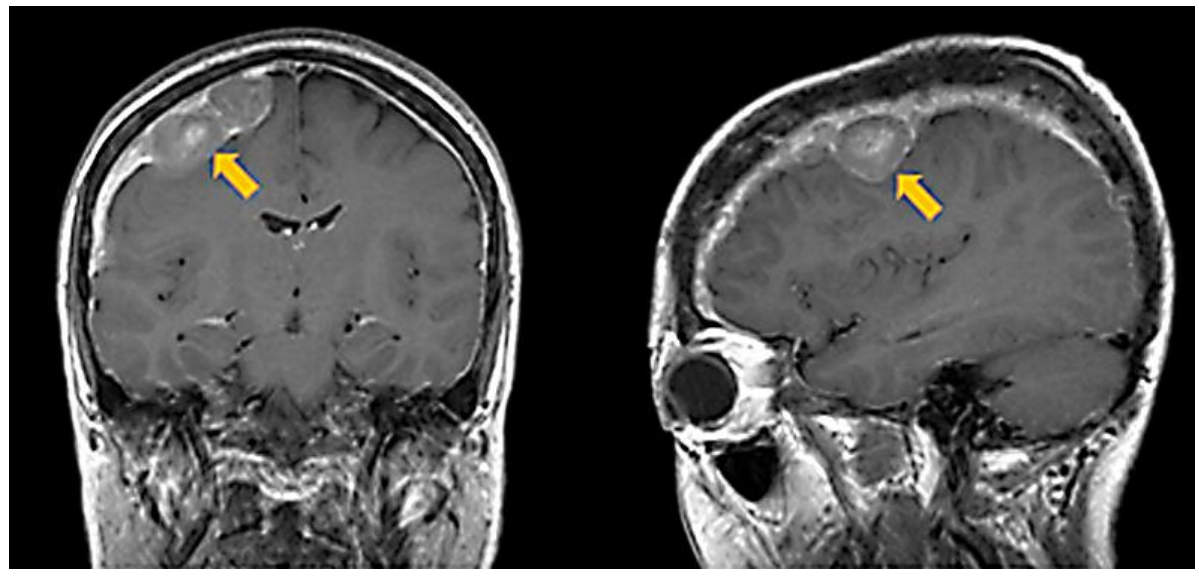

Fig. 1. MRI at diagnosis of MC. Bulky masses of $2.5 \times 1.9 \mathrm{~cm}$ in maximum size are located between the cranial bone and the cerebrum (arrows). 


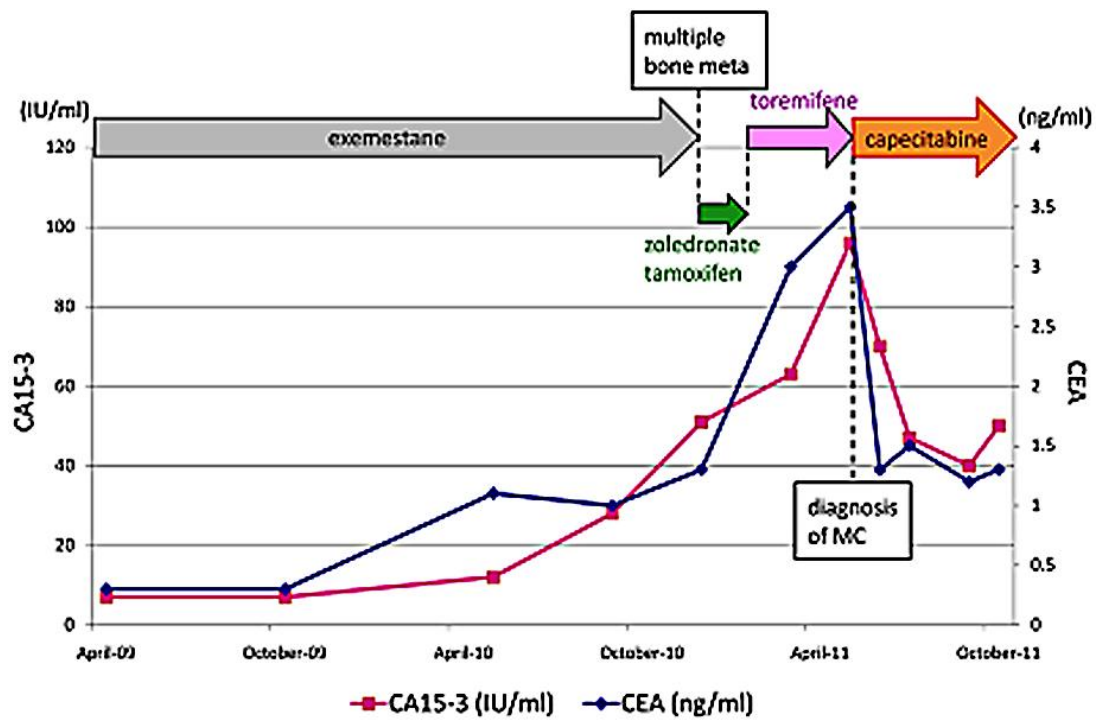

Fig. 2. Timelines of therapy and transition of tumor marker levels of CEA and CA15-3. After receiving capecitabine, both CEA and CA15-3 were markedly decreased.

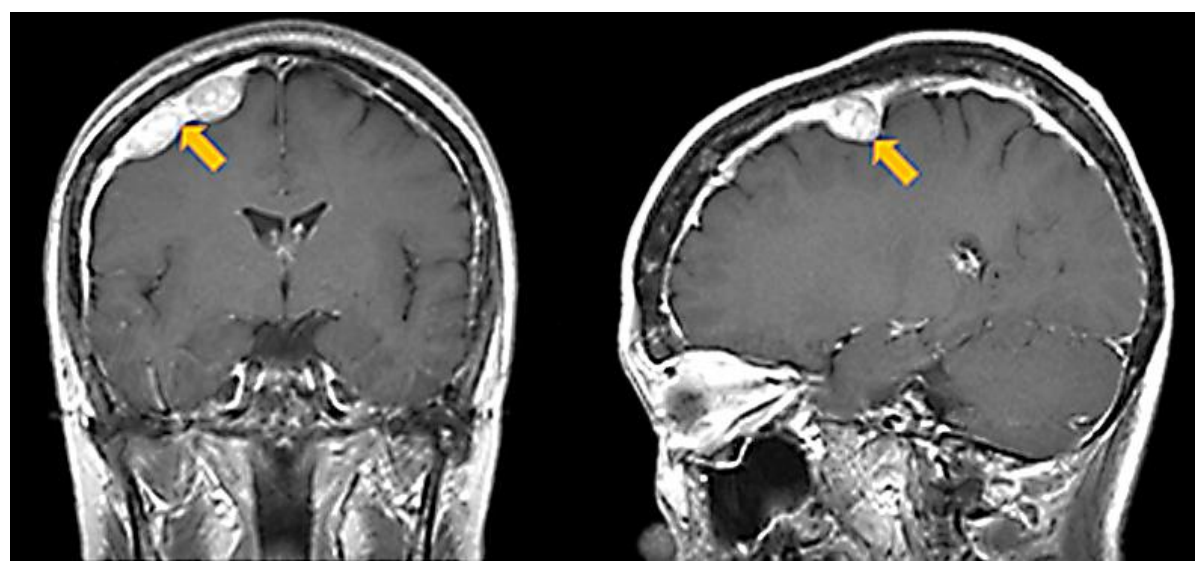

Fig. 3. MRI at about 2 months after the beginning of capecitabine monotherapy. Leptomeningeal masses have responded to capecitabine monotherapy. Maximum tumor size is $1.8 \times 1.0 \mathrm{~cm}$ (arrows). 


\section{References}

1 Boskovitz A, McLendon RE, Okamura T, Sampson JH, Bigner DD, Zalutsky MR: Treatment of HER2positive breast carcinomatous meningitis with intrathecal administration of alpha-particle-emitting (211)At-labeled trastuzumab. Nucl Med Biol 2009;36:659-669.

-2 Mustafa 0: Durable remission of leptomeningeal metastasis of breast cancer with letrozole: a case report and implications of biomarkers on treatment selection. J Clin Oncol 2003;33:229-231.

3 Kurosumi M, Akashi-Tanaka S, Akiyama F, Komoike Y, Mukai H, Nakamura S, et al: Histopathological criteria for assessment of therapeutic response in breast cancer (2007 version). Breast Cancer 2008;15:15-17.

4 Boogerd W, Dorresteijn LD, van Der Sande JJ, de Gast GC, Bruning PF: Response of leptomeningeal metastases from breast cancer to hormonal therapy. Neurology 2000;13:117-119.

5 Hattori S, Ogawa A, Oka H, Isogai Y, Inomata I: An autopsy case of meningeal carcinomatosis with vestibulocochlear nerve disturbance as the first manifestation. Gan No Rinsho 1986;32:1974-1980.

6 Gauthier H, Guilhaume MN, Bidard FC, Pierga JY, Girre V, Cottu PH, et al: Survival of breast cancer patients with meningeal carcinomatosis. Ann Oncol 2010;21:2183-2187.

7 Shoji O: A case of breast meningeal carcinomatosis. J Jpn Prac Surg Soc 1995;11:2340-2344.

8 Chamberlain MC: Response of leptomeningeal metastases from breast cancer to hormonal therapy. Neurology 2001;13:425-426.

-9 Rudnicha H, Niwinska A, Murauska M: Breast cancer leptomeningeal metastasis - the role of multimodality treatment. J Neurooncol 2007;84:57-62.

10 Oliveira M, Braga S, Passos-Coelho JL, Fonseca R, Oliveira J: Complete response in HER2+ leptomeningeal carcinomatosis from breast cancer with intrathecal trastuzumab. Breast Cancer Res Treat 2011;127:841-844.

11 Stemmler HJ, Mengele K, Schmitt M, Harbeck N, Laessig D, Herrmann KA, et al: Intrathecal trastuzumab (Herceptin) and methotrexate for meningeal carcinomatosis in HER2-overexpressing metastatic breast cancer: a case report. Anticancer Drugs 2008;19:832-836.

12 Rogers LR, Remer SE, Tejwani S: Durable response of breast cancer leptomeningeal metastasis to capecitabine monotherapy. Neuro Oncol 2004;6:63-64.

13 McEvoy GK (ed): Capecitabine; in AHFS Drug Information Bethesda, MD: American Society of HealthSystem Pharmacists, Inc., 2002, pp 916-921.

14 Heier MS, Heintz R, Fossa SD: Passage of 5'-dFUrd and its metabolites 5-FU and 5-FUH2 to CSF in a clinical phase 1 study. Acta Neurol Scand 1986;74:240-244.

15 Wang ML, Yung WK, Royce ME, Schomer DF, Theriault RL: Capecitabine for 5-fluorouracil-resistant brain metastases from breast cancer. Am J Clin Oncol 2001;24:421-424. 